\title{
Saúde Mental na Estratégia Saúde da Família: revisão da literatura brasileira
}

\author{
Mental Health in the Family Health Strategy: a review of \\ Brazilian literature
}

\author{
Luiz Gustavo Silva Souza \\ Doutor em Psicologia. \\ Endereço: Universidade Federal do Espírito Santo, Cemuni VI. \\ Programa de Pós-Graduação em Psicologia. \\ Endereço: Av. Fernando Ferrari, no 514, Goiabeiras, CEP 29075-910, \\ Vitória, ES, Brasil. \\ E-mail: luizsouzaœhotmail.com \\ Maria Cristina Smith Menandro \\ Doutora em Psicologia. Professora do Programa de Pós-Graduação \\ em Psicologia da Universidade Federal do Espírito Santo. \\ Endereço: UFES, Cemuni VI. Programa de Pós-Graduação em Psi- \\ cologia. Av. Fernando Ferrari, no 514, Goiabeiras, CEP 29075-910, \\ Vitória, ES, Brasil. \\ E-mail: crismenandro®uol.com.br

\section{Leandra Lúcia Moraes Couto} \\ Mestranda em Psicologia pelo Programa de Pós-Graduação em \\ Psicologia da Universidade Federal do Espírito Santo. \\ Endereço: Rua Professor Elpídio Pimentel, no 396, ap. 203, Jardim \\ da Penha, CEP 29060-170, Vitória, ES, Brasil. \\ E-mail: leandrabjळhotmail.com

\section{Polyana Barbosa Schimith} \\ Mestranda em Psicologia pelo Programa de Pós-Graduação em \\ Psicologia da Universidade Federal do Espírito Santo. \\ Endereço: Rua Alziro Zarur, no 330, ap. 307, Jardim da Penha, CEP \\ 29060-350, Vitória, ES, Brasil. \\ E-mail: ninha.bsøgmail.com

\section{Rebeca Panceri de Lima} \\ Graduanda em Psicologia pela Universidade Federal do Espírito \\ Santo. \\ Endereço: Rua Itapoã, no 26, Valparaíso, CEP 29175-821, Serra, \\ ES, Brasil. \\ E-mail: rebecapanceriøhotmail.com
}

\section{Resumo}

A Estratégia Saúde da Família orienta a organização da Atenção Primária à Saúde no Brasil e inclui importantes congruências programáticas com a Reforma Psiquiátrica. Os princípios de territorialização e acompanhamento longitudinal devem favorecer a construção de ações inovadoras de promoção, prevenção e reabilitação em saúde mental. O objetivo desta revisão foi analisar os principais temas da literatura científica brasileira sobre saúde mental na Estratégia Saúde da Família. Foram lidos os títulos de 267 artigos, publicados entre 1999 e 2009, e 38 artigos foram selecionados, segundo critérios específicos, para análise temática. A literatura abordou os temas da demanda em saúde mental, das percepções e práticas dos profissionais e da inserção do psicólogo na Atenção Primária. Os estudos revisados apontaram problemas como visões estereotipadas sobre os transtornos mentais, predominância da lógica manicomial, ausência de registros, fluxos, estratégias, apoio qualificado às famílias e de integração em rede. A meta-análise qualitativa indicou questões que podem fomentar o debate sobre o tópico e a realização de outras pesquisas, bem como a reflexão sobre a atuação profissional na interface entre Saúde Mental e Saúde da Família.

Palavras-chave: Atenção Primária à Saúde, Saúde Mental, Saúde da Família. 


\section{Abstract}

The Family Health Strategy establishes the principles of the Brazilian Primary Health Care and shares important goals with the Psychiatric Reform. The principles of territory-centered care and longitudinal care should enhance innovative actions of mental health promotion, prevention and rehabilitation. The aim of this review was to analyze the main themes approached by the Brazilian scientific literature concerning mental health in the Family Health Strategy. We read the titles of 267 articles published between 1999 and 2009. We followed specific criteria to select 38 articles for thematic analysis. The main themes were the demands in mental health, the perceptions and practices of health personnel and the role of the psychologist in Primary Care. The publications identified several problems: stereotypical views about mental disorders, the dominance of the hospitalization rationale, and the absence of clinical reports, strategies, qualified support to families and integrated health actions. The qualitative meta-analysis indicated questions that may strengthen the debate on the topic, the reflection on further research and on professional practice in the interface between Mental Health and Family Health.

Keywords: Primary Health Care; Mental Health; Family Health.

\section{Introdução}

Este artigo apresenta uma revisão da literatura brasileira sobre relações entre Estratégia Saúde da Família (ESF) e o campo da saúde mental. O procedimento de "revisão narrativa" (Rother, 2007) foi utilizado com o objetivo de identificar os principais núcleos temáticos abordados pela literatura científica vinculada a esses tópicos, bem como seus principais resultados e recomendações.

No quadro das diretrizes nacionais de saúde, a Saúde da Família é descrita como estratégia para organização de todo o sistema de Atenção Primária à Saúde (APS), que é, por sua vez, vista como porta de entrada do Sistema Único de Saúde (SUS) e como contato preferencial dos cidadãos com esse Sistema (Brasil, 2006).

As últimas quatro décadas foram de intensas transformações na assistência à saúde e à saúde mental. A Reforma Sanitária, com sua ênfase na APS, na defesa do controle social, da universalidade e da integralidade, apresenta compatibilidades evidentes com a Reforma Psiquiátrica, levando à necessidade de reflexão sobre práticas de saúde mental na APS. A Reforma Psiquiátrica brasileira é descrita como processo político-cultural, que envolve diversos atores sociais na transformação de saberes e práticas dirigidos aos transtornos mentais. As ações da APS, organizadas segundo a ESF, baseadas na criação de vínculos e no cuidado longitudinal, podem contribuir para construir alternativas às práticas de exclusão ou simples medicalização dos transtornos mentais (Brasil, 2005).

Amarante (2008) lembra que a Reforma Psiquiátrica não deve ser restrita à extinção física dos manicômios. Ela implica construir novas formas de lidar com as pessoas que estão em sofrimento mental. Para atingir os objetivos da Reforma, é necessário estar radicalmente próximo à população, promovendo vínculos duradouros, considerando a família como unidade de cuidados, conhecendo o território e inventando formas de intervir nele. Essas são justamente as potencialidades da ESF. Por isso, a produção de ações em saúde mental pelas equipes de ESF (e sua articulação em rede) pode ser vista como a forma mais promissora de mobilizar recursos comunitários e de concretizar a Reforma Psiquiátrica. 
(Amarante, 2008; Dalla Vecchia e Martins, 2009). A valorização de práticas de saúde mental na APS é fundamental para garantir a integralidade da atenção (Campos, 2003; Oliveira e col., 20o6; Tanaka e Ribeiro, 2009).

Esse princípio é defendido na proposta dos Núcleos de Apoio à Saúde da Família (NASF). Esses núcleos são constituídos por diferentes profissionais responsáveis por fornecer suporte a determinado número de equipes de Saúde da Família. Devem potencializar a resolutividade e a integralidade das ações dessas equipes. Sem ocupar a função de "porta de entrada", devem trabalhar segundo a lógica do apoio matricial. A Saúde Mental apresenta-se como uma das “áreas estratégicas" dos NASF (Brasil, 2009).

O estudo relatado neste artigo baseou-se em proposta semelhante àquela desenvolvida por Gama e Onocko Campos (2009). Em sua revisão, os autores mostraram que princípios importantes da Reforma Psiquiátrica foram incorporados nas reflexões sobre a APS, mas que evidências apontaram para a hegemonia do descompasso entre as diretrizes e a realidade dos serviços. Os autores consideraram a “Atenção Básica” em geral e revisaram periódicos de saúde coletiva. A revisão apresentada aqui se utilizou de procedimentos metodológicos diferentes (tendo coletado apenas dois artigos em comum), tratou especificamente da ESF e buscou artigos científicos em conjunto mais variado de periódicos. Os tópicos seguintes mostram que os resultados obtidos foram consoantes e complementares àqueles encontrados por Gama e Onocko Campos (2009).

\section{Procedimentos de Seleção da Literatura e de Análise}

A “revisão narrativa” visa a uma compreensão ampliada sobre o campo de estudos considerado (Rother, 2007), neste caso ESF e Saúde Mental. A revisão descrita aqui foi realizada por meio das bibliotecas eletrônicas Scientific Electronic Library Online (Scielo-Brasil) e Periódicos Eletrônicos em Psicologia (Pepsic). A escolha justifica-se por tratarem-se das duas mais importantes fontes abertas de literatura científica sobre o tema no País, contando com os principais periódicos nacionais de saúde coletiva, psicologia, psiquiatria e campos associa- dos. Por serem acervos de acesso livre, supõe-se que tenham boas possibilidades de impacto nos meios acadêmicos e profissionais.

A busca pelos artigos foi realizada no mês de janeiro de 2010 e considerou os trabalhos publicados de 1999 a 2009. Utilizou-se o "formulário básico" de busca, com os descritores "psf”, "programa saúde da família” e "esf”, “estratégia saúde da família”, buscados individualmente (um de cada vez) em "todos os índices" (all indexes). Com este procedimento, chegou-se ao total de 267 artigos, cujos títulos foram lidos. Foram selecionados os trabalhos que fizeram menção, no título, aos termos "saúde mental", "psicologia” e a termos associados. A menção desses termos no título foi considerada condição para o foco delimitado (relações entre ESF e saúde mental). A seleção identificou 38 artigos (24 em Scielo e 14 em Pepsic)

A análise foi feita por meio da leitura reiterada dos 38 artigos selecionados e de sua sistematização com um quadro específico. Esse quadro foi composto por colunas referentes à caracterização geral dos artigos (ano de publicação, metodologia etc.) e por colunas relativas a "principais resultados dos estudos" e "principais recomendações dos estudos". Os dados e análises obtidos são descritos a seguir.

\section{Resultados e Análises}

\section{Caracterização da literatura}

Dos artigos analisados $(n=38)$, contam-se 27 relatos de pesquisa, 6 relatos de intervenção e 5 ensaios teóricos. Dois deles foram publicados em inglês e todos os outros, em português. Não foi identificada qualquer publicação sobre o tema nos anos de 1999, 2000 e 2001. O número de publicações cresceu a partir de 2005, sinalizando aumento do interesse pelas relações entre ESF e saúde mental no período em questão.

Dentre os relatos de pesquisa $(n=27)$ foram encontrados 12 estudos com metodologia "quantitativa" e 15 com metodologia "qualitativa”. Os estudos considerados "quantitativos" priorizaram a apresentação dos resultados em números e análises estatísticas. Aqueles considerados "qualitativos" priorizaram a compreensão de sentidos e práticas, utilizando-se de variantes de análise de conteúdo 
ou análise de discurso. Os métodos de coleta mais utilizados nos estudos "quantitativos" foram questionários estruturados ( $n=8)$ e questionários de caracterização sociodemográfica $(n=6)$ e, nos “qualitativos", foram entrevistas semiestruturadas ( $\mathrm{n}=$ 10), observação participante $(n=5)$ e grupos focais $(n=4)$. É interessante ressaltar que a maior parte dos estudos não especificou a matriz teórica utilizada para análise dos dados. Assim como observado por Baptista e colaboradores (2009), alguns tomaram as atuais políticas de saúde como marco teórico.

\section{Análise temática}

Os artigos foram divididos em categorias temáticas de forma mutuamente excludente. Entretanto, é importante ressaltar que a inclusão de um artigo em uma categoria foi feita considerando a ênfase no tema correspondente e não sua abordagem exclusiva. Os tópicos que a literatura abordou foram seis: a) demandas em saúde mental dirigidas à APS; b) percepções e práticas dos profissionais quanto à saúde mental; c) relações entre CAPS (Centros de Atenção Psicossocial) e ESF; d) experiências dos agentes comunitários de saúde; e) famílias dos usuários e f) atuação do psicólogo na ESF.

\section{Demandas em saúde mental dirigidas à Atenção Primária à Saúde}

Estudos basearam-se direta ou indiretamente na noção de "transtornos mentais comuns" (TMCs), definidos como transtornos não psicóticos, que não se enquadram nas categorias formais dos manuais de diagnóstico, mas que já são fonte de disfunção ou incapacidade (Bandeira e col., 2007; Fortes e col., 2007; Gonçalves e Kapczinski, 2008a, 2008b; Maragno e col., 2006). Encontram-se nessa categoria principalmente quadros com sintomatologia de depressão, ansiedade e transtornos somatoformes. Essas pesquisas utilizaram-se de metodologia caracteristicamente quantitativa e buscaram avaliar a prevalência de TMCs.

Embora com alguma variação, as prevalências encontradas foram altas, em torno de 30\%. Uma das pesquisas incluiu entrevista psiquiátrica padronizada com 754 participantes e verificou que em cerca de $51 \%$ dos casos estudados, os usuários apresentaram um ou mais diagnósticos psiquiátricos (Gonçalves e Kapczinski, 2008a). Mostrou-se que as maiores prevalências foram encontradas em mulheres, pessoas com menor escolaridade e em pessoas com baixa renda, incluindo desempregados. Levantou-se a hipótese de que a opressão de gênero, a exclusão e as injustiças sociais são elementos relevantes para explicar essa associação (Maragno e col., 2006).

Depressão e ansiedade surgiram como os sintomas mais comuns, sendo também frequentes transtornos somatoformes e dissociativos, com alta taxa de comorbidade. A pesquisa de Cruz e colaboradores (2005) identificou alta prevalência de depressão puerperal $(37,1 \%)$ em mulheres atendidas por Unidades de Saúde da Família (USF). Dentre os idosos pesquisados por Floriano e Dalgalarrondo (2007), cerca de um terço obteve escores para transtornos mentais. Os transtornos estiveram significativamente associados à incapacidade funcional e ao morar sozinho (falta de suporte social).

A ESF tem teoricamente maior potencialidade para lidar com esses problemas do que a APS convencional. Entretanto, em uma pesquisa, a prevalência dos transtornos não foi significativamente menor em áreas atendidas por ESF se comparadas com outras áreas (Maragno e col., 2006). Pode-se supor que, embora a ESF traga inovações em termos de estrutura e processo de trabalho, ela ainda não foi capaz de tocar no centro dos fatores que transformariam a atenção em saúde mental, tais como a maior e melhor disponibilidade de profissionais e de recursos, a estruturação de fluxos próprios para a saúde mental, a escuta psicossocial, o acolhimento da alteridade e o trabalho integrado em rede. Outros estudos apoiaram a hipótese de que a ESF ainda não contemplou esses fatores de forma consistente.

Os autores foram unânimes em considerar que o campo da saúde mental deve receber maior atenção por parte da APS. Gonçalves e Kapczinski (2008a) sugeriram que esse campo seja eleito como prioridade, assim como o são a diabetes e a hipertensão. Maragno e e colaboradores (2006) lembraram que os transtornos mentais dizem respeito diretamente à adesão aos tratamentos de saúde e à qualidade de vida. Recomenda-se que o fluxo interno da USF preveja um caminho específico para as questões de saúde mental (Maragno e col., 2006; Bandeira e col., 2007), evitando que elas se diluam no conjunto das muitas outras atividades da Unidade. Gonçalves e 
Kapczinski (2008a) sugeriram adotar questionários de rastreamento na rotina de trabalho. Bandeira e colaboradores (2007) e Fortes e e colaboradores (2007) enfatizaram a necessidade de capacitação (permanente) das equipes de APS, enfocando especialmente ansiedade, depressão e transtornos somatoformes.

\section{Percepções e práticas dos profissionais quanto à saúde mental}

Diante da demanda descrita, o que a ESF tem feito? Um conjunto de estudos buscou explorar percepções e práticas de profissionais no campo da saúde mental. Utilizaram delineamento caracteristicamente qualitativo, o que dificulta a generalização dos resultados, mas permite formular insights importantes sobre o tema. É interessante notar que esses estudos, assim como aqueles dos três tópicos seguintes, focaram práticas e saberes dirigidos aos transtornos psicóticos, em contraste com os levantamentos descritos acima.

Dois estudos associados (Nunes e col., 2007; Jucá e col., 2009) investigaram como profissionais de uma equipe de saúde da família compreendiam os transtornos mentais. Os profissionais apontaram causas muito variadas para o sofrimento mental. Apresentaram desencontros significativos nas opiniões sobre transtornos com maior prevalência. A “depressão" foi o transtorno mais citado, porém houve menções a autismo e psicose, o que mostra descompasso entre as opiniões dos profissionais e os dados dos estudos de prevalência descritos acima. Além disso, não houve menções a alcoolismo e uso de drogas. Souza (2005) abordou a identificação de problemas relacionados ao uso de álcool, analisando dados do Ministério da Saúde. $\mathrm{O}$ autor verificou que a população adscrita à ESF aumentou bastante nos últimos quinze anos, mas a proporção de registros de casos de "alcoolismo" diminuiu de $1,4 \%$ para $0,82 \%$.

Nunes e colaboradores (2007) e Jucá e colaboradores (2009) também recolheram dados sobre como profissionais desenvolviam intervenções em saúde mental. Notaram ausência de registro em prontuários; dificuldades de estabelecer vínculos com os usuários; temor da equipe quanto à violência do bairro e quanto a possíveis comportamentos agressivos dos usuários; permanência de referências "biologizantes" para compreensão do sofrimento mental; permanência de atitudes moralizantes e repressivas; ausência de estratégias para lidar com violência e com problemas decorrentes do consumo de álcool e outras drogas e permanência da lógica da internação como horizonte "terapêutico". Além disso, profissionais declararam que se sentiam despreparados para lidar com práticas de saúde mental e que as capacitações oferecidas pelos gestores eram insuficientes, gerando sentimentos de impotência e frustração (Nunes e col., 2007; Jucá e col., 2009). Resultados semelhantes foram obtidos por Lucchese e colaboradores (2009).

Silva e colaboradores (2005) mostraram que enfermeiros construíam concepções de cuidado ligadas ao modelo tradicional de atenção à saúde, mescladas com novos enfoques em saúde coletiva. Porém, não havia definição clara das ferramentas de intervenção que poderiam utilizar em saúde mental. Souza e colaboradores (2007) entrevistaram 134 enfermeiros e verificaram que a maior parte (97\%) declarou não ter formação específica em saúde mental. A atividade que prevalecia era o encaminhamento (72,3\%), seguido pelo atendimento médico (47,7\%). O serviço mais procurado era o hospital psiquiátrico (87,7\%), seguido pelo ambulatório (42,3\%) e o menos procurado era o CAPS-ad (19,2\%) (vale lembrar que os CAPSs são descritos pelas diretrizes como peçaschave da rede - Brasil, 2005). Nesse estudo, cerca de $15 \%$ dos enfermeiros afirmaram que sua equipe não tinha qualquer atuação em saúde mental.

Práticas das USFs e dos profissionais também foram pesquisadas indiretamente, pela abordagem de usuários e familiares. Usuários e familiares investigados por Koga e colaboradores (2006) declararam que a ESF facilitou a marcação de consultas. Entretanto, houve evidências de que as orientações sobre medicação psicotrópica, transmitidas por agentes comunitários de saúde, não eram satisfatórias e de que essas orientações não eram fornecidas por outros membros das equipes. As intervenções dos profissionais pareciam produzir poucas transformações nas relações entre famílias e usuários. Dentre os usuários investigados por Dimenstein e colaboradores (2005), selecionados pelos critérios de uso de psicotrópicos e de internação prévia em hospital psiquiátrico, havia demanda reprimida, com muitas pessoas sem acompanhamento (cerca de 30\% dos 
entrevistados); uso indiscriminado de benzodiazepínicos; uso da medicação como principal alternativa de tratamento; alto índice de internações (40,7\% já haviam sido internados) e uso pouco frequente do CAPS. Avaliou-se que a atenção à saúde mental continuava medicalizada e hospitalocêntrica.

Ribeiro e colaboradores (2009) compararam unidades com e sem equipes de ESF e verificaram que as unidades com equipe, em geral, não produziram resultados significativamente melhores, apresentando deficiência em registros e altas taxas de abandono de tratamento.

Diante dos dados, a sugestão mais frequente da literatura analisada foi fornecer mais e melhor capacitação dos profissionais. Segundo os autores, essa capacitação deveria ser permanente; baseada em reuniões periódicas com um supervisor proveniente de CAPS; deveria não ser restrita a aspectos técnicos, incluindo reflexões epistemológicas capazes de elucidar as relações entre loucura e sociedade; deveria ter como objetivo compartilhar e reconstruir ideias, experiências, estratégias e conceitos, permitindo que os profissionais formulem sistemas de significados, instrumentos de intervenção e projetos em comum.

É curioso, portanto, notar que a abordagem direta de estratégias de capacitação dos profissionais para lidar com questões de saúde mental foi escassa na literatura analisada. O único trabalho desse tipo tematizou o alcoolismo: diagnóstico e intervenção breve (Corradi-Webster e col., 2005). Foram pesquisadas as percepções de profissionais sobre um treinamento para atenção a alcoolistas (é importante registrar que o treinamento em si não foi descrito). As percepções dos profissionais foram em geral positivas. Dentre os efeitos do treinamento, estiveram a diminuição de estereótipos e preconceitos e a adoção de estratégias preventivas e adaptadas ao universo psicossocial do usuário. $\mathrm{Na}$ literatura pesquisada, havia escassez de relatos sobre métodos para realizar a capacitação dos profissionais e de dados objetivos sobre a efetividade dessas capacitações.

\section{Relações entre CAPS e ESF}

Alguns estudos enfocaram a produção conjunta da atenção em saúde mental entre CAPS e ESF. 0 apoio matricial fornecido por CAPS foi descrito como forma de promover o envolvimento dos profissionais desses diferentes níveis, não se tratando de simples supervisão de casos. 0 apoio matricial pode ser percebido de forma positiva pelos profissionais, entendido como "regulador de fluxo", definindo em qual nível cada caso será acompanhado (USF ou CAPS). Entretanto, também pode ser considerado pelas equipes como sobrecarga de trabalho (Bezerra e Dimenstein, 2008). Os estudos identificaram empecilhos para a implantação do apoio matricial: falta de capacitação específica para saúde mental, pequeno número de profissionais, falta de articulação entre eles, e baixa remuneração.

Os autores avaliaram que a articulação entre os CAPS e a rede de APS é ainda precária e que os serviços disponíveis se apresentam dispersos e isolados. Recomendaram o esclarecimento do papel do CAPS no SUS, junto aos profissionais, e a construção efetiva de uma rede de serviços. Os profissionais deveriam refletir continuamente sobre a experiência de matriciamento, para permanecerem abertos à inovação (Bezerra e Dimenstein, 20o8; Delfini e col., 2009).

Para Vieira Filho e Nóbrega (2004), as conexões entre CAPS, ESF e outras instituições que compõem os recursos do território devem funcionar como espaço dinâmico de continência do sofrimento mental. Delfini e colaboradores (2009) destacam que o apoio matricial, baseado em reuniões e visitas domiciliares, pode enriquecer a prática cotidiana e transformar a percepção dos profissionais acerca das pessoas portadoras de transtorno mental, desfazendo preconceitos. Destacam ainda que esses efeitos podem ser particularmente importantes para os agentes comunitários de saúde.

\section{As experiências de agentes comunitários de saúde}

Agentes de saúde pesquisados por Barros e colaboradores (2009) forneceram respostas heterogêneas sobre transtornos mentais, com raciocínios frequentemente restritos ao aspecto biológico. As práticas relatadas foram caracterizadas por distanciamento cognitivo, afetivo e físico. 0 isolamento social, característica comum em transtornos mentais, foi interpretado unicamente como decorrência de fatores individuais e não sociais. Os usuários eram percebidos como instáveis, potencialmente agressivos e perigosos, o que acarretava sentimento de medo 
(Barros e col., 2009). As agentes pesquisadas por Campos e Romagnoli (2007) também apresentaram dificuldades para se relacionar com a alteridade: condutas estereotipadas, medo do diferente, postura assistencialista, entre outras.

As práticas "centradas na doença”, como acompanhamento de medicação e consultas, foram mais citadas. Mas também houve referências a práticas mais atentas à promoção de saúde, como construção de vínculos, participação em grupos terapêuticos, ênfase na interação social, apoio à família e visitas domiciliares (Barros e col., 2009). Os agentes pesquisados por Feneric e colaboradores (2004) mostraram-se inseguros, por exemplo, sobre o que fazer diante de uma "descompensação" emocional de um usuário. Em geral, eles se percebiam como importantes para as terapêuticas empregadas, mas tinham dificuldades em explicar casos bem-sucedidos, o que mostra que os instrumentos de intervenção e seus "princípios ativos" não estavam claros.

Ao mesmo tempo, Barros e colaboradores (2009) notaram que os agentes se apropriaram de noções importantes da Reforma Psiquiátrica, como a ênfase na importância das interações sociais para a reabilitação psicossocial. Os agentes fazem parte da população atendida, o que pode facilitar a postura de escuta e o diálogo, instrumentos de trabalho muitas vezes preteridos pelo "especialista" (Barros e col., 2009). Os autores ressaltaram a importância dos agentes para a implantação dos cuidados em saúde mental na APS e foram unânimes ao recomendar que eles recebam mais e melhor capacitação Recomendou-se ainda que as equipes valorizem o saber dos agentes e os vejam como atores do processo de construção da ESF e da Reforma Psiquiátrica, tomando por base o pressuposto de que eles criam efetivamente, apesar das dificuldades, novas formas de lidar com os transtornos mentais (Feneric e col., 2004; Barros e col., 2009).

\section{As famílias dos usuários}

Como os profissionais percebem as famílias dos usuários portadores de transtornos mentais? Souza e Scatena (2005) realizaram pesquisa com duas equipes de ESF, principalmente agentes comunitários. Verificaram que as famílias eram vistas simultaneamente como cuidadoras e alvos de cuidados em saúde; simultaneamente motivadoras e sofredoras de preconceitos; frequentemente impotentes, carentes de recursos (inclusive psicológicos) e produtoras de maus tratos. Os profissionais pareciam não saber lidar com o sofrimento dos familiares que cumpriam funções de cuidador. Diante disso, os autores repetiram a frequente recomendação da literatura para capacitar os profissionais. Sugeriram também que as equipes adotem uma concepção ampliada de família, o que pode aumentar os recursos comunitários para a reabilitação psicossocial.

Severo e colaboradores (2007) entrevistaram 121 familiares-cuidadores e verificaram que cerca de $70 \%$ eram mulheres. De forma geral, a experiência de cuidar era entendida como tarefa constante e difícil, envolvendo desgaste físico e emocional, podendo gerar sobrecarga na rotina familiar, sobrecarga financeira, adoecimento da cuidadora, levando a possíveis internações do usuário. Os autores avaliaram que o suporte emocional fornecido às cuidadoras pode melhorar o tratamento e sugeriram que a família deve ser inserida efetivamente no projeto terapêutico, baseando-se no pressuposto de que as relações familiares e sociais são importantes agentes terapêuticos ao lado da medicação psiquiátrica.

\section{A atuação do psicólogo na Estratégia Saúde da Família}

Os estudos que trataram da atuação do profissional de psicologia junto a equipes de ESF foram de três tipos: estudos dedicados a fornecer bases teóricas para a atuação do psicólogo, pesquisas empíricas que buscaram verificar como se dá essa atuação e relatos de intervenção.

Os ensaios teóricos foram unânimes ao propor a inserção efetiva do psicólogo nas equipes de ESF. França e Viana (2006) argumentaram que os princípios do SUS implicam a adoção de concepção complexa de saúde, que deve incluir a preocupação com seus determinantes psicológicos e sociais. Os relatos sistematizados por Soares (2005) mostram que há boas experiências de inserção de psicólogos em equipes de ESF e que esses profissionais têm sido importantes para a promoção de autonomia e de adesão ao tratamento. Outra possibilidade teórica é apresentada por Franco e Bastos (2002), que veem a psicologia de Bronfenbrenner como referencial promissor. Camargo-Borges e Cardoso (2005) recomendaram a adoção da psicologia social da saúde 
como referencial teórico. Segundo as autoras, esse referencial possui importantes pontos de contato com a ESF, como a ênfase na produção coletiva da saúde, na interação dos atores, na promoção de vínculo e de acolhimento; o objetivo de mobilização dos coletivos para transformação das condições sociais e sanitárias e para a promoção de cidadania.

As pesquisas empíricas verificaram que o profissional de psicologia é considerado importante pelas equipes de ESF, sendo visto como referência para a área de saúde mental. Os profissionais ouvidos por Bittencourt e Mateus (2006) consideraram que o psicólogo poderia desenvolver atividades junto a grupos específicos (diabéticos etc.), realizar visitas domiciliares, participar de "todas as atividades da Unidade" e orientar a equipe quanto a questões técnicas e também quanto a seu próprio funcionamento interno. Souza e Carvalho (2003) e Clemente e colaboradores (2008), pesquisando a atuação de acadêmicos e residentes de psicologia, observaram características semelhantes a essas expectativas. Esses autores foram unânimes ao recomendar a inserção e a consolidação da psicologia na ESF. Clemente e colaboradores (2008) recomendaram ainda a reformulação do Sistema de Informações Ambulatoriais (SIA-SUS), que se deveria adequar às características da atuação dos psicólogos.

Em outros trabalhos, um dos obstáculos para a efetiva integração entre psicologia e ESF ficou evidente: características da formação em psicologia. Os sentidos construídos pelas psicólogas entrevistadas por Costa e Olivo (2009) em torno de sua prática eram centrados, em geral, no modelo psicológico-clínico tradicional. Ao mesmo tempo, reivindicavam inserção efetiva na equipe e trabalho interdisciplinar, incluindo parceria com agentes comunitários e abordagem das angústias da própria equipe. Oliveira e colaboradores (2007) perceberam grande indefinição quanto à inserção da psicologia na ESF tanto para os profissionais em geral quanto para os próprios psicólogos, com hegemonia do "modelo clínico tradicional”. Em consonância com esse debate, os relatos de intervenção preocuparam-se em mostrar que a inserção da psicologia na ESF pode se dar além do modelo tradicional, "de consultório". o modelo tradicional é por vezes esperado (e demandado) pelo resto da equipe (Gama e Koda, 2008; Soares e Pinto, 2008), mas caberia aos psicólogos mostrar outros caminhos.

A variedade de estratégias de intervenção relatada foi realmente grande: acolhimento, oficinas psicossociais, grupos operativos, grupos de mediação, grupos de psicoterapia, de psicoterapia breve, atendimento familiar, educação em saúde na sala de espera etc. (Cardoso, 2002; Gama e Koda, 2008; Cardoso e Féres-Carneiro, 20o8; Soares e Pinto, 2008). Cardoso (2002) e Cardoso e Féres-Carneiro (2008) notaram que, com as atividades grupais, os usuários mostraram maior disponibilidade para falar de si, para expressar sentimentos e interagir com os demais participantes dos grupos. Entretanto, também foram descritas situações em que os usuários apresentaram dificuldades para participar de grupos (Gama e Koda, 2008), sendo recomendada a manutenção de estratégias de atendimento individual. Os autores dos relatos de intervenção também recomendaram a inserção e o fortalecimento da psicologia na ESF, a ampliação das estratégias de atuação, com adequação às demandas da ESF, e o rearranjo da formação dos psicólogos, que tem que ser mais atenta às questões de saúde coletiva.

O Quadro 1 esquematiza os resultados desta revisão narrativa, organizando os estudos segundo os seis temas identificados. São fornecidos dados sobre tipo de método adotado nos estudos e sobre a meta-análise qualitativa efetuada nesta revisão.

A descrição dos tipos de método adotados (predominantemente "quali” ou "quanti”) contribui para caracterizar o campo de pesquisas em questão. Ressalta-se a predominância de relatos de intervenção e ensaios teóricos no Tema 6, possivelmente decorrentes do esforço de buscar justificação teórico-prática para a presença da psicologia na ESF. Os principais resultados obtidos e recomendações feitas nos estudos são resumidos. A coluna "Questões e possíveis lacunas" propõe reflexões críticas sobre o material revisado na forma de perguntas, apontando possíveis objetos de interesse para estudos futuros. Sem a pretensão de fornecer respostas, espera-se que essas perguntas fomentem o debate em um campo de pesquisa e de atuação caracterizado como "recente". Do ponto de vista profissional, esse debate pode ser especialmente relevante para profissionais integrantes dos NASF. 


\section{Quadro I - Sistematização dos resultados da revisão segundo temas encontrados e respectiva meta-análise qualitativa}

\begin{tabular}{|c|c|c|c|}
\hline \multirow{2}{*}{ Temas e estudos } & \multirow{2}{*}{$\begin{array}{c}\text { Metodologia } \\
\text { ou tipo }\end{array}$} & \multicolumn{2}{|l|}{ Meta-análise qualitativa } \\
\hline & & Principais resultados e recomendações & Questões e possíveis lacunas \\
\hline \multicolumn{2}{|l|}{ Tema I: Demandas em saúde mental } & \multirow{8}{*}{$\begin{array}{l}\text { Alta prevalência de "transtornos } \\
\text { mentais comuns" (principalmente } \\
\text { sintomas de ansiedade e depressão) } \\
\text { e de depressão puerperal. Associação } \\
\text { com opressão de gênero e exclusão } \\
\text { social. Recomendações: saúde mental } \\
\text { como prioridade; questionários/ } \\
\text { triagem na rotina das USFs. }\end{array}$} & \multirow{8}{*}{$\begin{array}{l}\text { Como explicar a "produção social" } \\
\text { dos TMCs? Quais os significados } \\
\text { sociais das altas prevalências } \\
\text { de ansiedade e depressão? Que } \\
\text { recursos da USF mobilizar para } \\
\text { implantar a triagem e melhorar a } \\
\text { "escuta" dirigida ao sofrimento } \\
\text { mental? }\end{array}$} \\
\hline Bandeira e colaboradores (2007) & Quanti & & \\
\hline Cruz e colaboradores (2005) & Quanti & & \\
\hline Floriano e Dalgalarrondo (2007) & Quanti & & \\
\hline Fortes e colaboradores (2007) & Quanti & & \\
\hline Gonçalves e Kapczinski (2008a) & Quanti & & \\
\hline Gonçalves e Kapczinski (2008b) & Quanti & & \\
\hline Maragno e colaboradores (2006) & Quanti & & \\
\hline \multicolumn{2}{|c|}{ Tema 2: Percepções e práticas dos profissionais } & \multirow{11}{*}{$\begin{array}{l}\text { Persistência de crenças e percepções } \\
\text { tradicionais sobre a loucura. } \\
\text { Hegemonia de práticas de exclusão. } \\
\text { Pouca atenção a problemas com álcool } \\
\text { e outras drogas. } \\
\text { Recomendação de ampliar e melhorar } \\
\text { a capacitação dos profissionais. }\end{array}$} & \multirow{11}{*}{$\begin{array}{l}\text { Como concretizar em métodos } \\
\text { os princípios apresentados para } \\
\text { a capacitação dos profissionais? } \\
\text { Como avaliar a eficácia das } \\
\text { capacitações? Os pesquisadores } \\
\text { podem construir estratégias de } \\
\text { assistência em conjunto com os } \\
\text { profissionais? }\end{array}$} \\
\hline Corradi-Webster e colaboradores (2005) & $\mathrm{RI}$ & & \\
\hline Dimenstein e colaboradores (2005) & Quanti & & \\
\hline Jucá e colaboradores (2009) & Quali & & \\
\hline Koga e colaboradres (2006) & Quanti & & \\
\hline Lucchese e colaboradores (2009) & Quali & & \\
\hline Nunes e colaboradores (2007) & Quali & & \\
\hline Ribeiro e colaboradores (2009) & Quanti & & \\
\hline Silva e colaboradores (2005) & Quali & & \\
\hline Souza (2005) & Quanti & & \\
\hline Souza e colaboradores (2007) & Quanti & & \\
\hline \multicolumn{2}{|l|}{ Tema 3: Relações entre CAPS e ESF } & \multirow{4}{*}{$\begin{array}{l}\text { Apoio matricial como forma de } \\
\text { compartilhar a produção da saúde } \\
\text { mental. Relações em geral ainda } \\
\text { distantes entre CAPS e ESF. }\end{array}$} & \multirow{4}{*}{$\begin{array}{l}\text { Quais soluções os atores têm } \\
\text { encontrado para a questão sobre } \\
\text { que serviço gerencia os casos, CAPS } \\
\text { ou USF? O que determina essas } \\
\text { soluções? }\end{array}$} \\
\hline Bezerra e Dimenstein (2008) & Quali & & \\
\hline Delfini e colaboradores (2009) & $\mathrm{RI}$ & & \\
\hline Vieira Filho e Nóbrega (2004) & ET & & \\
\hline \multicolumn{2}{|c|}{ Tema 4: Experiências dos agentes de saúde } & \multirow{4}{*}{$\begin{array}{l}\text { Agentes com crenças e práticas } \\
\text { tradicionais, mas também com } \\
\text { maior proximidade aos usuários. } \\
\text { Recomendações: maior capacitação } \\
\text { e maior inclusão de seu saber nas } \\
\text { equipes. }\end{array}$} & \multirow{4}{*}{$\begin{array}{l}\text { Que fatores impedem a auto e } \\
\text { hetero afirmação dos agentes como } \\
\text { protagonistas da reabilitação } \\
\text { psicossocial? Como lidar com esses } \\
\text { fatores? }\end{array}$} \\
\hline Barros e colaboradores (2009) & Quali & & \\
\hline Campos e Romagnoli (2007) & Quali & & \\
\hline Feneric e colaboradores (2004) & Quali & & \\
\hline \multicolumn{2}{|l|}{ Tema 5: As famílias dos usuários } & \multirow{3}{*}{$\begin{array}{l}\text { Importância das(os) cuidadoras(es) } \\
\text { para reabilitação psicossocial. } \\
\text { Ausência de estratégias sistemáticas } \\
\text { para apoiá-las. }\end{array}$} & \multirow{3}{*}{$\begin{array}{l}\text { Como aprimorar a "escuta" } \\
\text { ao sofrimento comum entre } \\
\text { as cuidadoras? Que fatores } \\
\text { psicossociais (estereótipos, crenças } \\
\text { etc.) agem na produção desse } \\
\text { sofrimento? }\end{array}$} \\
\hline Severo e colaboradores (2007) & Quali & & \\
\hline Souza e Scatena (2005) & Quali & & \\
\hline
\end{tabular}



Quadro I - Sistematização dos resultados da revisão segundo temas encontrados e respectiva meta-análise
qualitativa (continuação)

\begin{tabular}{|c|c|c|c|}
\hline \multirow{2}{*}{ Temas e estudos } & \multirow{2}{*}{$\begin{array}{c}\text { Metodologia } \\
\text { ou tipo }\end{array}$} & \multicolumn{2}{|l|}{ Meta-análise qualitativa } \\
\hline & & Principais resultados e recomendações & Questões e possíveis lacunas \\
\hline \multicolumn{2}{|c|}{ Tema 6: A atuação do psicólogo na ESF } & \multirow{14}{*}{$\begin{array}{l}\text { Importância da inserção efetiva do } \\
\text { psicólogo na ESF. Posição privilegiada } \\
\text { para promoção de adesão e cidada- } \\
\text { nia. Modelo clínico tradicional como } \\
\text { obstáculo. Pluralidade de alternativas } \\
\text { já testadas qualitativamente. Reco- } \\
\text { mendações de adequar a formação do } \\
\text { psicólogo, de consolidar sua atuação } \\
\text { em ESF, valorizando formas próprias } \\
\text { de registro de produção. }\end{array}$} & \multirow{14}{*}{$\begin{array}{l}\text { É possível construir parâmetros } \\
\text { comuns para atuação do psicólogo } \\
\text { em ESF, apesar da diversidade } \\
\text { teórica da psicologia? Se sim, } \\
\text { quais? É possível avaliar a eficácia } \\
\text { dos tipos de intervenção descritos? } \\
\text { Como? }\end{array}$} \\
\hline Bittencourt e Mateus (2006) & Quali & & \\
\hline Camargo-Borges e Cardoso (2005) & ET & & \\
\hline Cardoso (2002) & $\mathrm{RI}$ & & \\
\hline Cardoso e Féres-Carneiro (2008) & RI & & \\
\hline Clemente e colaboradores (2008) & Quali & & \\
\hline Costa e Olivo (2009) & Quali & & \\
\hline França e Viana (2006) & ET & & \\
\hline Franco e Bastos (2002) & ET & & \\
\hline Gama e Koda (2008) & $\mathrm{RI}$ & & \\
\hline Oliveira e colaboradores (2007) & Quali & & \\
\hline Soares (2005) & ET & & \\
\hline Soares e Pinto (2008) & $\mathrm{RI}$ & & \\
\hline Souza e Carvalho (2003) & Quali & & \\
\hline
\end{tabular}

Legenda: $\mathrm{ET}=$ ensaio teórico; $\mathrm{RI}=$ Relato de intervenção.

\section{Considerações Finais}

Em resumo, a literatura analisada abordou os temas das prevalências de transtornos mentais, das percepções e experiências de profissionais (incluindo agentes de saúde) sobre temas como loucura, CAPS, familiares-cuidadores e da atuação do psicólogo na ESF. Por um lado, registrou-se intensa demanda em saúde mental advinda de usuários e de seus familiares e/ou cuidadores. Por outro, verificaram-se percepções e práticas, histórica e contextualmente arraigadas, que atuam como obstáculos para a resposta efetiva a essa demanda.

Assim como na revisão feita por Gama e Onocko Campos (2009), constatou-se persistência do paradigma biomédico tradicional, pouca correspondência entre as diretrizes de inclusão da saúde mental e a realidade dos serviços, dificuldades na relação entre equipes de ESF e CAPS. Mas, as ausências não foram absolutas. Estudos mostraram que profissionais construíam concretamente formas de lidar com os problemas de saúde mental na APS mais adequadas aos princípios do SUS e da Reforma Psiquiátrica. Entretanto, essas estratégias permaneciam "obscuras" (os próprios profissionais não tinham clareza sobre elas) assistemáticas e desarticuladas da rede de serviços. Isso sugere que a atenção à saúde mental na APS brasileira do início do século XXI ainda se encontra em seus "tateios" iniciais. Mas, verificou-se aumento da produção sobre o tema no período considerado (resultado também condizente com Gama e Onocko Campos, 2009). 0 meio acadêmico brasileiro parece responder a desafios atuais e importantes, acompanhando o desenvolvimento da APS no País.

Os resultados desta revisão corroboram a avaliação de Tanaka e Ribeiro (2009) de que “[...] a inclusão efetiva de assistência à saúde mental na atenção básica ainda é uma realidade pouco frequente" (p. 479). No entanto, existem bons trabalhos, que apontam alternativas operacionais promissoras (por exemplo, Oliveira e col., 2006; Onocko Campos e Gama, 2008; Dalla Vecchia e Martins, 2009, além dos trabalhos que compuseram esta revisão). A inclusão das ações de saúde mental na APS deve ter o objetivo de promover a autonomia dos usuários, seu autocuidado e suas relações familiares e sociais, contemplando a atenção a transtornos de diferentes graus de severi- 
dade e persistência. Para concluir, cabe reconhecer que o desafio acadêmico e profissional que se impõe é difícil, mas também estimulante.

\section{Referências}

AMARANTE, P. D. C. Saúde mental, desinstitucionalização e novas estratégias de cuidado. In: GIOVANELLA, L. et al (Orgs.). Políticas e sistema de saúde no Brasil. Rio de Janeiro:

Fiocruz, 2008. p. 735-759.

BANDEIRA, M.; FREITAS, L. C.; CARVALHO FILHO, J. G. T. Avaliação da ocorrência de transtornos mentais comuns em usuários do Programa de Saúde da Família. Jornal Brasileiro de Psiquiatria, Rio de Janeiro, v. 56, n.1, p.41-47, 2007.

BAPTISTA, T. W. F.; FAUSTO, M. C. R.; CUNHA, M. S. Análise da produção bibliográfica sobre atenção primária à saúde no Brasil em quatro periódicos selecionados. Physis: Revista de Saúde Coletiva, Rio de Janeiro, v. 19, n. 14, p. 1007-1028, 2009.

BARROS, M. M. M.; CHAGAS, M. I. O.; DIAS, M. S. A. Saberes e práticas do agente comunitário de saúde no universo do transtorno mental. Revista Ciência \& Saúde Coletiva, Rio de Janeiro, v. 14, n. 1, p. 227-232, 2009.

BEZERRA, E.; DIMENSTEIN, M. Os CAPS e o trabalho em rede: tecendo o apoio matricial na atenção básica. Psicologia Ciência e Profissão, Brasília, v. 28, n. 3, p. 632-645, 2008.

BITTENCOURT, R. A. A.; MATEUS, M. L. F. Possibilidades de atuação do psicólogo no programa saúde da família: a experiência de Bonito-MS. Psicologia Ciência e Profissão, Brasília, v. 26, n. 2, p. 328-343, 2006.

BRASIL. Ministério da Saúde. Secretaria de Atenção à Saúde. Departamento de Atenção Básica. Política Nacional de Atenção Básica. Brasília, DF, 2006.

BRASIL. Ministério da Saúde. Secretaria de Atenção à Saúde. Coordenação Geral de Saúde Mental. Reforma psiquiátrica e política de saúde mental no Brasil: documento apresentado à Conferência Regional de Reforma dos Serviços de Saúde Mental: 15 anos depois de Caracas. OPAS. Brasília, DF, 2005.
BRASIL. Ministério da Saúde. Secretaria de Atenção à Saúde. Departamento de Atenção Básica. Cadernos de atenção básica. Diretrizes do NASF, Núcleos de Apoio à Saúde da Família. Versão preliminar. Brasília, DF, 2009.

CAMARGO-BORGES, C.; CARDOSO, C. L. A psicologia e a estratégia saúde da família: compondo saberes e fazeres. Psicologia e Sociedade, Florianópolis, v. 17, n. 2, p. 26-32, 2005.

CAMPOS, A. P.; ROMAGNOLI, R. C. Os encontros entre os agentes comunitários de saúde e as famílias dos portadores de transtorno. Mental, Barbacena, v. 5, n. 9, p. 79-99, 2007.

CAMPOS, G. W. S. Saúde paidéia. São Paulo: Hucitec, 2003.

CARDOSO, C. L.; FÉRES-CARNEIRO, T. Sobre a família: com a palavra, a comunidade. Estudos e Pesquisas em Psicologia, Rio de Janeiro, v. 8, n. 2, p. 523-539, 2008.

CARDOSO, C. L. A inserção do psicólogo no Programa Saúde da Família. Psicologia Ciência e Profissão, Brasília, v. 22, n. 1, p. 2-9, 2002.

CLEMENTE, A. et al. Residência multiprofissional em saúde da família e a formação de psicólogos para a atuação na atenção básica. Saúde e Sociedade, São Paulo, v. 17, n. 1, p. 176-184, 2008.

CORRADI-WEBSTER, C. M. et al. Capacitação de profissionais do programa de saúde da família em estratégias de diagnóstico e intervenções breves para o uso problemático de álcool. SMAD: Revista Eletrônica Saúde Mental Álcool e Drogas, Ribeirão Preto, v. 1, n. 1, p.1-10, 2005.

COSTA, D. F. C.; OLIVO, V. M. F. Novos sentidos para a atuação do psicólogo no Programa Saúde da Família. Revista Ciência \& Saúde Coletiva, Rio de Janeiro, v. 14, sup. 1, p. 1385-1394, 2009.

CRUZ, E. B. S.; SIMÕES, G. L.; FAISAL-CURY, A. Rastreamento da depressão pós-parto em mulheres atendidas pelo Programa de Saúde da Família. Revista Brasileira de Ginecologia e Obstetrícia, Rio de Janeiro, v. 27, n. 4, p. 181-188, 2005 . 
DALLA VECCHIA, M.; MARTINS, S. T. F.

Desinstitucionalização dos cuidados a pessoas com transtornos mentais na atenção básica: aportes para a implementação de ações. Interface: comunicação, saúde, educação, Botucatu, v. 13, n. 28, p. 151-164, 2009.

DELFINI, P. S. S. et al. Parceria entre CAPS e PSF: o desafio da construção de um novo saber. Revista Ciência \& Saúde Coletiva, Rio de Janeiro, v. 14, sup. 1, p. 1483-1492, 2009.

DIMENSTEIN, M. et al. Demanda em saúde mental em unidades de saúde da família. Mental, Barbacena, v. 3, n. 5, p. 23-41, 2005.

FENERIC, S. R. G.; PEREIRA, W. A. B.; ZEOULA, F. M. Saúde mental e programa de saúde da família: percepções do agente comunitário de saúde.

Psicologia Hospitalar, São Paulo, v. 2, n. 2, p. o-o, 2004.

FLORIANO, P. J.; DALGALARRONDO, P. Saúde mental, qualidade de vida e religião em idosos de um Programa de Saúde da Família. Jornal Brasileiro de Psiquiatria, Rio de Janeiro, v. 56, n. 3, p. 162-170, 2007.

FORTES, S.; VILLANO, L. A. B.; LOPES, C. S. Nosological profile and prevalence of common mental disorders of patients seen at the Family Health Program (FHP) units in Petrópolis, Rio de Janeiro. Revista Brasileira de Psiquiatria, São Paulo, v. 3o, n. 1, p. 32-37, 2007.

FRANÇA, A. C. P.; VIANA, B. A. Interface psicologia e programa saúde da família - PSF: reflexões teóricas. Psicologia Ciência e Profissão, Brasília, v. 26, n. 2, p. 246-257, 2006.

FRANCO, A. L. S.; BASTOS, A. C. S. Um olhar sobre o Programa de Saúde da Família: a perspectiva ecológica na psicologia do desenvolvimento segundo Bronfenbrenner e o modelo da vigilância da saúde. Psicologia em Estudo, Maringá, v. 7, n. 2, p. 65-72, 2002.

GAMA, C. A. P.; KODA, M. Y. Psicologia comunitária e programa de saúde da família: relato de uma experiência de estágio. Psicologia Ciência e Profissão, Brasília, v. 28, n. 2, p. 418-429, 2008.
GAMA, C. A. P.; ONOCKO CAMPOS, R. Saúde mental na atenção básica - uma pesquisa bibliográfica exploratória em periódicos de saúde coletiva (1997-2007). Cadernos Brasileiros de Saúde Mental, Florianópolis, v. 1, n. 2, p. 112-131, 2009.

GONÇALVES, D. M.; KAPCZINSKI, F. Prevalência de transtornos mentais em indivíduos de uma unidade de referência para Programa Saúde da Família em Santa Cruz do Sul, Rio Grande do Sul, Brasil. Cadernos de Saúde Pública, Rio de Janeiro, v. 24, n. 9, p. 2043-2053, 2008a.

GONÇALVES, D. M.; KAPCZINSKI, F. Transtornos mentais em comunidade atendida pelo Programa Saúde da Família. Cadernos de Saúde Pública, Rio de Janeiro, v. 24, n. 7, p. 1641-165o, 20o8b.

JUCÁ, V. J. S.; NUNES, M. O.; BARRETO, S. G. Programa de Saúde da Família e saúde mental: impasses e desafios na construção da rede.

Revista Ciência \& saúde coletiva, Rio de Janeiro, v. 14, n. 1, p. 173-182, 2009.

KOGA, M.; FUREGATO, A. R. F.; SANTOS, J. L. F. Opinions of the staff and users about the quality of the mental health care delivered at a family health program. Revista Latino-Americana de Enfermagem, Ribeirão Preto, v. 14, n. 2, p.163-169, 2006.

LUCCHESE, R. et al. Saúde mental no Programa Saúde da Família: caminhos e impasses de uma trajetória necessária. Cadernos de Saúde Pública, Rio de Janeiro, v. 25, n. 9, p. 2033-2042, 2009.

MARAGNO, L. et al. Prevalência de transtornos mentais comuns em populações atendidas pelo Programa Saúde da Família (QUALIS) no município de São Paulo, Brasil. Cadernos de Saúde Pública, Rio de Janeiro, v. 22, n. 8, p. 16391648, 2006.

NUNES, M.; JUCÁ, V. J.; VALENTIM, C. P. B. Ações de saúde mental no Programa Saúde da Família: confluências e dissonâncias das práticas com os princípios das reformas psiquiátrica e sanitária. Cadernos de Saúde Pública, Rio de Janeiro, v. 23, n. 10, p. 2375-2384, 2007. 
OLIVEIRA, A. G. B.; VIEIRA, M. A. M.; ANDRADE, S. M. R. Saúde mental na saúde da família: subsídios para o trabalho assistencial. São Paulo: Olho d'água, 2006.

OLIVEIRA, I. F.; SILVA, F. L.; YAMAMOTO, O. H. A psicologia no Programa de Saúde da Família (PSF) em Natal: espaço a ser conquistado ou um limite da prática psicológica? Aletheia, Canoas, n. 25, p. 05-19, 2007.

ONOCKO CAMPOS, R.; GAMA, C. Saúde mental na atenção básica. In: CAMPOS, G. W. S.; GUERRERO, A. V. P. (Orgs.). Manual de práticas de atenção básica: saúde ampliada e compartilhada. São Paulo: Hucitec, 2008. p. 221-246.

RIBEIRO, M. S. et al. Comparação da assistência em saúde mental em unidades básicas de saúde com ou sem equipe do Programa de Saúde da Família. Revista de Psiquiatria do Rio Grande do Sul, Porto Alegre, v. 31, n. 1, p. 40-50, 2009.

ROTHER, E. T. Editorial. Revisão sistemática X revisão narrativa. Acta paulista de enfermagem, São Paulo, v. 20, n. 2, p. 5-6, 2007.

SEVERO, A. K. S. et al. A experiência de familiares no cuidado em saúde mental. Arquivos Brasileiros de Psicologia, Rio de Janeiro, v. 59, n. 2, p. 143-155, 2007.

SILVA, A. T. M. C. et al. A saúde mental no PSF e o trabalho de enfermagem. Revista Brasileira de Enfermagem, Brasília, v. 58, n. 4, p. 411-415, 2005.

SOARES, N. M.; PINTO, M. E. B. Interfaces da Psicologia aplicada à saúde: atuação da Psicologia na estratégia Saúde da Família em Londrina. Revista da SBPH, Belo Horizonte, v. 11, n. 2, p. 89$100,2008$.
SOARES, T. C. "A vida é mais forte do que as teorias” o psicólogo nos serviços de atenção primária à saúde. Psicologia Ciência e Profissão, Brasília, v. 25, n. 4, p. 590-601, 2005.

SOUZA, A. J. F. et al. A saúde mental no Programa de Saúde da Família. Revista Brasileira de Enfermagem, Brasília, v. 6o, n. 4, p. 391-395, 2007.

SOUZA, M. L. P. Expansão do Programa Saúde da Família (PSF) e identificação de problemas relacionados ao uso de álcool no Brasil. Revista Brasileira de Psiquiatria, São Paulo, v. 27, n. 4, p. 342-343, 2005.

SOUZA, R. A.; CARVALHO, A. M. Programa de Saúde da Família e qualidade de vida: um olhar da psicologia. Estudos de Psicologia, Natal, v. 8, n. 3 , p. 515-523, 2003.

SOUZA, R. C.; SCATENA, M. C. M. Produção de sentidos acerca da família que convive com o doente mental. Revista Latino-Americana de Enfermagem, Ribeirão Preto, v. 13, n. 2, p. 173-179, 2005 .

TANAKA, O. Y.; RIBEIRO, E. L. Ações de saúde mental na atenção básica: caminho para ampliação da integralidade da atenção. Revista Ciência \& Saúde Coletiva, Rio de Janeiro, v. 14, n. 2, p. 477-486, 2009.

VIEIRA FILHO, N. G.; NÓBREGA, S. M. A atenção psicossocial em saúde mental: contribuição teórica para o trabalho terapêutico em rede social. Estudos de Psicologia, Natal, v. 9, n. 2, p. 373-379, 2004 .

Recebido em: 30/10/2010

Reapresentado em: 04/06/2012

Aprovado em: 16/09/2012 